\title{
CEREBROVASCULAR DISEASE IN ANTIPHOSPHOLIPID SYNDROME: 20 year experience in a University Hospital
}

\author{
V. Ros-Castelló ${ }^{1}$, A. Gómez-López ${ }^{1}$, E. Natera ${ }^{1}$, A. Sánchez-Sánchez ${ }^{1}$, J. Martínez-Poles ${ }^{1}$, A. Cruz Culebras ${ }^{1}$, R. Vera ${ }^{1}, A$. \\ DeFelipe ${ }^{1}$, C. Matute ${ }^{1}$, R. Álvarez-Velasco ${ }^{1}$, A. Alonso-Cánovas ${ }^{1}$, J. Masjuan ${ }^{1}$. \\ ${ }^{1}$ Ramón y Cajal University Hospital, Stroke Unit, Neurology, Madrid, Spain.
}

INTRODUCTION: Antiphospholipid syndrome (APS) is an autoimmune disorder for which optimal clinical management remains controversial. Specific immunologic profiles such as lupic anticoagulant (LA) positivity or triple antiphospholipid antibodies (aPL) positivity may have prognostic relevance.

METHODS: Retrospective analysis of clinical, immunological and therapeutic variables of patients with APS related cerebrovascular disease from 1997 to 2017.

\section{RESULTS}

Seventeen patients (82\% female, mean age of $48 \pm 17$ years) were included.

Eight patients (47\%) were receiving antithrombotic therapy because of previous thrombosis.

Three patients had secondary APS.

$\begin{array}{ll}\text { Baseline characteristics } & \\ \text { Mean age, years (SD) } & 48(17) \\ \text { Female, } \mathrm{n}(\%) & 14(82) \\ \text { Primary APS, } \mathrm{n}(\%) & 14(82) \\ \text { Secondary APS, } \mathrm{n}(\%) & 3(18) \\ \text {-Systemic lupus erythematosus } & 2 \\ \text {-Eosinophilic granulomatosis with } & 1 \\ \text { polyangiitis } & \\ \text { Previous thrombotic events: } & \\ \text {-Ischemic stroke, } \mathrm{n} & 5 \\ \text {-TIA, } \mathrm{n} & 1 \\ \text {-Other arterial thrombosis, } \mathrm{n} & 2 \\ \text {-Venous thrombosis, } \mathrm{n} & 3 \\ \text {-Obstetric APS, } \mathrm{n} & 3 \\ \text { Previous antithrombotic treatment: } & \\ \text {-Antiplatelets, } \mathrm{n} & 3 \\ \text {-Vitamin K antagonists, } \mathrm{n} & 2 \\ \text {-Direct oral anticoagulants, } \mathrm{n} & 1 \\ \text {-Antiplatelets+ vitamin } \mathrm{K} \text { antagonists, } \mathrm{n} & 1 \\ \text {-Subcutaneous heparin, } \mathrm{n} & 1 \\ \end{array}$

Image 1. MRI venography showing left transverse sinus thrombosis presented by one patient.

\begin{tabular}{ll|}
\hline Clinical and therapeutic variables & \\
Cerebrovascular disease: & 12 \\
-Ischemic stroke, $\mathrm{n}$ & 4 \\
-TIA, $\mathrm{n}$ & 1 \\
-CVT, $\mathrm{n}$ & \\
Treatment on discharge: & 4 \\
-Antiplatelets, $\mathrm{n}$ & 9 \\
-Vitamin K antagonists, $\mathrm{n}$ & 2 \\
-Antiplatelets+ vitamin $\mathrm{K}$ antagonists, $\mathrm{n}$ & 1 \\
-Antiplatelets direct oral anticoagulants, $\mathrm{n}$ & \\
&
\end{tabular}

Twelve patients presented with ischemic stroke, 4 patients with transient ischemic Neither triple aPL nor LA positivity were attack (TIA) and 1 patient with cerebral significantly associated with stroke/TIA venous thrombosis (CVT). Clinical recurrence or death. management was heterogeneous.

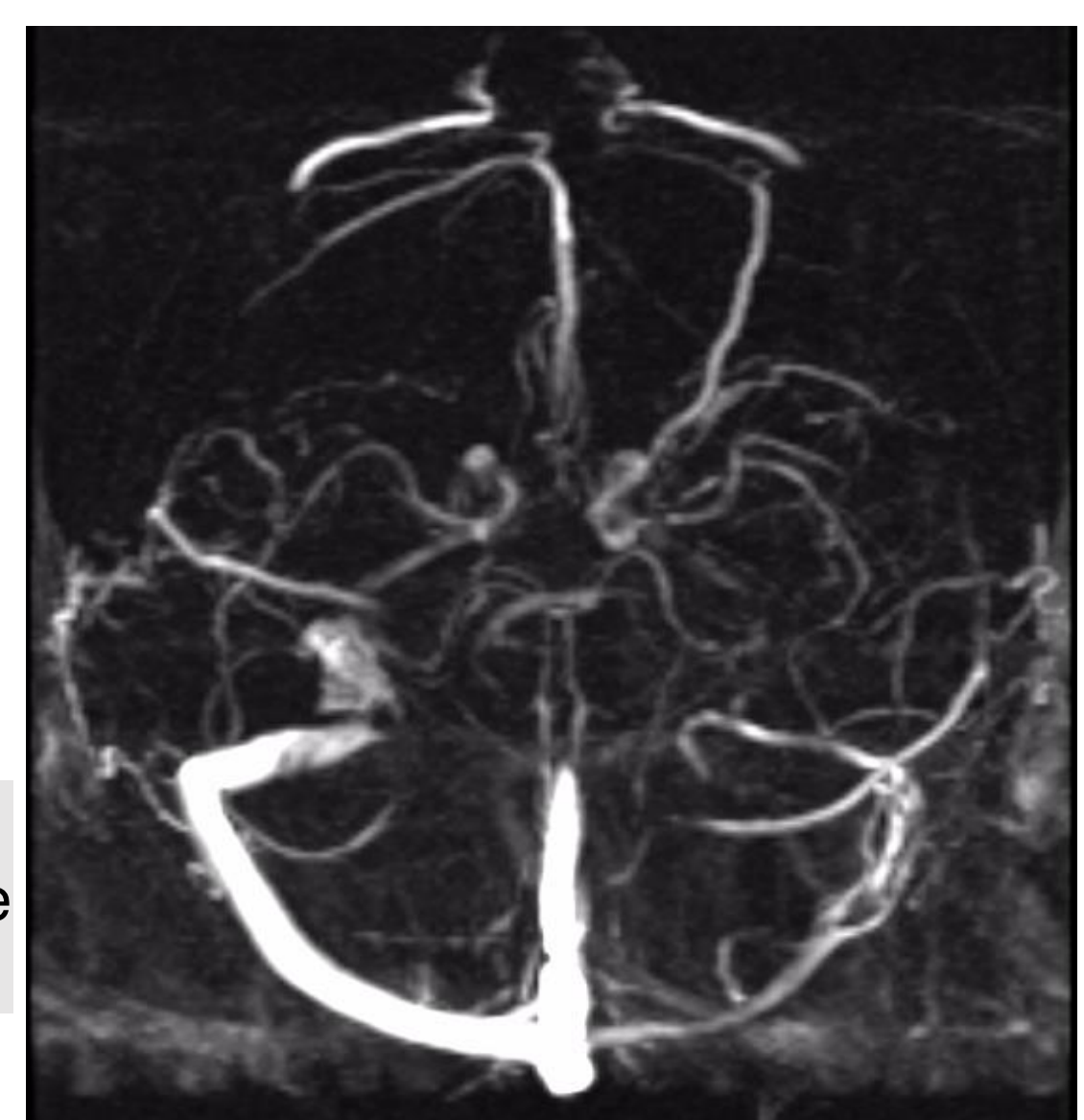

Immunologic profile

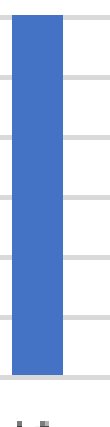

LA ACL IgM aCL IgG aB2GP II aB2GP' I Triple APL

$\begin{array}{ll}\text { Prognostic variables } & \\ \text { Mean follow-up, years (SD) } & 4.9(4.5) \\ \text { mRS end of follow-up: } & \\ -0-2, n & 9 \\ -3-5, n & 3 \\ -6, n & 5 \\ \text { Causes of death: } & 2 \\ \text {-Intracerebral haemorrhage, } \mathrm{n} & 1 \\ \text {-Multiple ischemic stroke, } \mathrm{n} & 1 \\ \text {-Catastrophic APS, } \mathrm{n} & 1 \\ \text {-Unkown, } \mathrm{n} & \\ \text { Long-term recurrences: } & 2 \\ \text {-TIA, } \mathrm{n} & 1 \\ \text {-Stroke, } \mathrm{n} & 1 \\ \text {-Other arterial thrombosis, } \mathrm{n} & \\ & \\ \text { APS related cerebrovascular disease had } \\ \quad \text { significant morbidity and mortality. }\end{array}$

DISCUSSION: Evidence regarding optimal antithrombotic management in patients with cerebrovascular disease due to APS is lacking. Either antiplatelet therapy and vitamin K antagonists (at an INR target 2-3 or $>3$ ) or its combination have been proposed. Direct oral anticoagulants have been used in some patients. LA and triple APL positivity have been related with a greater risk of arterial and venous thrombosis and, consequently, with a poorer prognosis.

CONCLUSIONS: In our experience, APS related cerebrovascular disease had significant morbidity and mortality. Clinical management was heterogeneous. No predictors of poor prognosis were detected, although small sample size may be responsible.

Ruiz-Irastorza G, Crowther M, Branch W, Khamashta MA. Antiphospholipid syndrome. Lancet. 2010 Oct 30; 376:1498-509

Espinosa G, Cervera R. Current treatment of antiphospholipid syndrome: lights and shadows. Nat Rev Rheumatol. 2015

Oct; $11: 586-96$.

Lim W, Crowther MA, Eikelboom JW. Management of antiphospholipid antibody syndrome: a systematic review. JAMA 2006; 295: $1050-57$

Kernan W, Ovbiagele B, Black H, Bravata D, Chimowitz M, Ezekowitz M et al. Guidelines for the prevention of stroke in patients with stroke and transient ischemic attack: a guideline for healthcare professionals from the American Heart 